\title{
ASTROMETRY WITH SMALL TELESCOPES
}

\author{
W. S. Penhallow \\ Physics Department \\ University of Rhode Island \\ Kingston, Rhode Island, 02881 \\ U. S. A.
}

ABSTRACT. At the Quonochontaug Observatory of the University of Rhode Island, we specialize in the development of simple seeing limited optical telescopes. Our main instrument is a heavy Serrier trussed $f / 10.7$, . 4-m astrometric reflector which provided the last-minute astrometry in the successful prediction of the occultation of SAO 120774 by Herculina in 1978. It is similar in design to the U. S. Naval Observatory 1.5-m reflector. Its scale of $47.1 \mathrm{H} / \mathrm{mm}$ makes it potentially more powerful than the Lick .5-m, f/7 astrograph. However, its field of view of $1.3^{\circ}$ $\mathrm{x} 1.7^{\circ}$ is insufficient to capture enough AGK3R reference stars which are needed to consistently provide tenth-of-a-second-of-arc accuracy. To solve this problem, a $.24-.32-\mathrm{m}, \mathrm{f} / 6.3$ lensless Schmidt was developed to provide a $3.8^{\circ}$ field at $135^{\prime \prime} / \mathrm{mm}$ and is mounted on the $.4-\mathrm{m}$ telescope. It can be used by itself and/or to provide a secondary reference frame for the main instrument. The measures from the plates and film are reduced by a suite of interactive computer programs which allows one to choose the proper reduction model based on the Eichhorn criterion. Over 100 positions of stars, asteroids, comets and supernovae have been provided with these instruments, and a regular observing program including participation in the IHW is underway.

\section{RESULTS}

1.1. Table I - Residuals for Comet Observations (Astrometric Reflector)

$\begin{array}{lrrrr} & \text { 1978 U.T. } & \frac{\Delta \alpha \cos \delta}{" \prime} & \frac{\Delta \delta}{1 "} \\ \text { Wild 2 (1978b) } & \text { Feb.9.08 } & +0.8 & +0.6 \\ & 10.11 & -0.3 & +0.8 \\ 12.08 & -0.5 & 0.0 \\ \text { Meier (1978f) } & \text { May 3.12 } & \underline{+0.7} & {[\underline{+2.1]} \text { [disc.] }} \\ & & \text { RMS } & 0.6 & 0.6\end{array}$


1.2. Table II - Residuals for P/Giacobini-Zinner (Schmidt)

\begin{tabular}{|c|c|c|c|}
\hline \multirow{7}{*}{ P/Giacobini-Zinner } & 1985 U.T. & $\Delta \alpha \cos \delta$ & $\Delta \delta$ \\
\hline & July 24.19955 & $\begin{array}{l}\text { ". } \\
+.77\end{array}$ & -1.24 \\
\hline & 25.25833 & -.45 & -.33 \\
\hline & Aug. $\quad 3.25833$ & $-1 \cdot 33$ & +1.15 \\
\hline & 5.26771 & {$[-5.21][$ disc. $]$} & -1.30 \\
\hline & 7.21965 & +.32 & -.60 \\
\hline & 18.34722 & +1.55 & -.73 \\
\hline & & .99 & .96 \\
\hline
\end{tabular}

1.3. Table III -Internal Residuals for Amphitrite (Schmidt)

\begin{tabular}{|c|c|c|c|}
\hline \multirow{3}{*}{ Amphitrite } & 1985 U.T. & $\Delta \alpha \cos \delta$ & $\frac{\Delta \delta}{11}$ \\
\hline & $\begin{array}{r}\text { June } 21.08057 \\
21.08542 \\
21.09167\end{array}$ & $\begin{array}{l}+0.07 \\
-0.12 \\
+0.05 \\
\end{array}$ & $\begin{array}{l}-0.17 \\
+0.30 \\
-0.13 \\
\end{array}$ \\
\hline & & $\begin{array}{l}0.08 \\
0.05\end{array}$ & $\begin{array}{l}0.21 \\
0.12\end{array}$ \\
\hline
\end{tabular}

1.4. Table IV - A Star That Might Be Occulted by P/Halley (Schmidt)

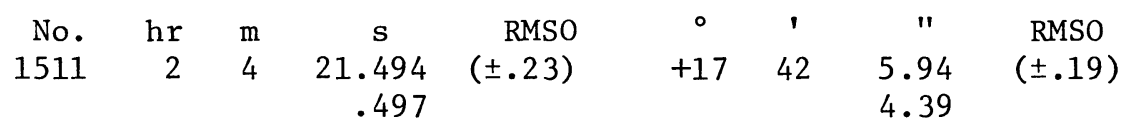

\section{CONCLUSIONS}

It has been demonstrated that simple reflecting telescopes of small size and low cost can be used to make useful astrometric observations when suitable measuring engines are available. The rapid turn-around time, particularly with asteroid, comet and planetary occultations, provides for a powerful means of evaluating computer errors, reduction models, telescope optics, measuring techniques, star catalog positions, and general procedures. Accuracies better than 0:"1 can be obtained with the $.4-\mathrm{m}$ (16-inch), f/10.7 astrometric reflector with four exposures under favorable conditions. Corresponding accuracies for the .24-.32-m, f/6.3 lensless Schmidt are about 0":2. A linear six constant reduction model is adequate to attain the latter accuracy with the Schmidt when the field is kept to $3: 8$.

\section{RECOMMENDATIONS}

Occultations of stars by solar system bodies have become a very active and fruitful field of endeavor. The need for more astrometry is pressing. It is recommended that (a) more observatories, both professional and amateur, be encouraged to attempt this type of work; (b) a survey be made of the old and little-used measuring engines that might be made available for possible long-term loan to observatories interested in doing this type of work; (c) that some type of amateur-professional organization be formed to guide and nurture the reawakening of the ancient art of doing good astrometry. 